\title{
Study on Tensile Behavior of Natural Fiber Reinforced PP Composites
}

\author{
Xianyi Shao ${ }^{1, \mathrm{a}}$, Luju $\mathrm{He}^{2, \mathrm{~b},{ }^{*}}, \mathrm{Li} \mathrm{Ma}^{3, \mathrm{c}}$ \\ ${ }^{1}$ College of Physics \& Electronic Engineering, Taizhou University, Taizhou 318000, China \\ ${ }^{2}$ Education and Information Technology Center, Guangdong University of Petrochemical \\ Technology, Maoming 525000, Guangdong, China \\ ${ }^{3}$ College of Mechanical and Electrical Engineering, Guangdong University of Petrochemical \\ Technology, Maoming 525000, Guangdong, China \\ asxy8718@163.com, bheluju@gdupt.edu.cn, 'cmali@gdupt.edu.cn
}

\begin{abstract}
Keywords: Natural fiber; Surface modification; Mechanical behavior; Composite
Abstract. Cellulose (CE), sawdust (SD) and wheat straw (WS) were subjected to triethoxysilane (AS) and methacriloxy propyl trimethoxy silane (MS), and maleic anhydride grafted polypropylene (MAPP) to improve interfacial bonding with matrix. Composites were prepared by these fibers as reinforcement. Tensile tests results show that composites were significantly enhanced by employment of coupling agents and MAPP to be the most effective coupling agent. Mechanical properties of SD composites exhibit the best performance compared to CE and WS. Optimum conditions for coupling agents were found to be $1 \mathrm{wt} \%$ for silane coupling agents and $5 \mathrm{wt} \%$ for MAPP.
\end{abstract}

\section{Introduction}

Natural fibers based polymer composites have received considerable attentions, particularly in automotive industries and construction due to their advantages of low density, acceptable specific strength, less tool wear, biodegradability, and renew-ability ${ }^{[1]}$. However, natural fiber are hydrophilic and they are not well compatible with hydrophobic polymer matrices such as polyethylene (PE) and polypropylene (PP) leading to weak fiber-matrix interfacial adhesion and poor mechanical properties of the composites ${ }^{[2-3]}$. Different approaches have been applied to change the fiber/matrix adhesive properties in natural fiber-reinforced composites: chemical or physical modifications of matrix, fiber or both the composites ${ }^{[4-5]}$. In this work, the effect of interfacial modification including triethoxysilane (AS) and methacriloxy propyl trimethoxy silane (MS), and maleic anhydride grafted polypropylene (MAPP) on tensile properties of cellulose (CE), sawdust (SD) and wheat straw (WS) was investigated. In addition, the influence of fiber content on the properties of the composites was examined.

\section{Experimental}

Isotactic PP in pellet form was used as the polymeric matrix material. Cellulose (CE), sawdust (SD) and wheat straw (WS) were used as fiber. Silane coupling agents and MAPP were used as suitable candidates to alter incompatibility between fiber and matrix. Chopped dried short untreated or treated fibers and PP pellets were compounded with fiber content of 10, 20, 30 and $40 \mathrm{wt} \%$ by using ThermoPrism twin screw extruder for good mixing of fiber and polymer. Mechanical properties were determined by tensile test at room temperature and at least five specimens for each composite formulation were tested.

\section{Results and Discussion}

Tensile Strength of Composites. Fig. 1 demonstrates that tensile strength of CE, SD and WS loaded composites as a function of fiber loading. It was obtained that tensile strength decreased with increasing fiber loading irrespective of fiber type. At 40wt\% fiber loading, the decline was 41.9, 42.4 and $44.4 \%$ for CE, SD and WS/PP composites, respectively. The decline in tensile strength with 
increasing fiber content is the usual case for natural fiber reinforced thermoplastics ${ }^{[6]}$. Table.1 shows increase in tensile strength of $30 \mathrm{wt} \%$ loaded composites with surface treatment. As a general trend, AS and MS treatments provided maximum tensile strength increase at $1 \mathrm{wt} \%$ silane treatment with respect to fiber weight. Tensile strength increase between 6 and $11.6 \%$ could be achieved at $1 \mathrm{wt} \%$ level of silane treatment. Silane treatment above $1 \%$ decreased tensile strength of composites to the levels of untreated composites. In silane treatment, the formation of silanol as a result of hydrolysis and the respective siloxane as an effect of condensation was to be expected. This should favor the formation of hydrogen bonds with the hydroxyl groups that are on the surface of cellulose. Probably, siloxane chains were formed and they become so excessively long that suffered crosslinking reactions and therefore, the formation of a weak interface ${ }^{[7]}$.Similarly, optimum condition for MAPP was 5wt \% with respect to polypropylene content. It was also observed that MAPP provided a much more efficient reinforcement compared to two types of silane treatments. At optimum conditions, MAPP had increased tensile strength 32.6, 50.4 and $49.4 \%$ for CE, SD and WS composites, respectively. The increase in tensile strength with silane treatment at optimum conditions did not exceed 14.2\%. Fig. 2 illustrates the effect of coupling agents on tensile strength of CE, SD and WS/PP composites at 30wt\% loading. This graph shows that MAPP has a great coupling efficiency compared to AS and MS. Another conclusion is MAPP is more effective coupling agent for SD/PP composites since increase in tensile strength is much more pronounced for SD/PP composites compare to two other composite systems. Although 30\% fiber was employed, there was almost no tensile strength decrease for SD/PP composites compared to neat PP.

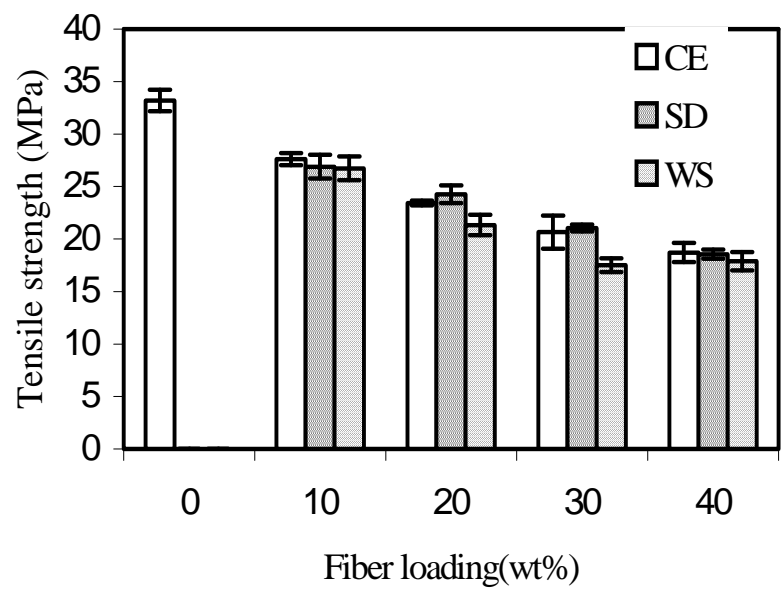

Fig.1. Effect of fiber loading on tensile strength of PP/C, SD and WS composites

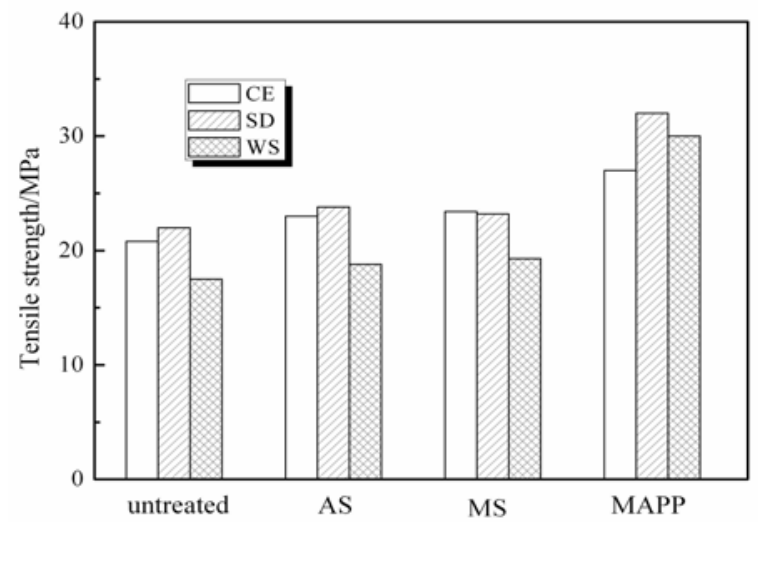

Fig.2. Effect of coupling agent on tensile strength of PP/CE, SD and WS composites

Table.1 tensile strength with varying treatment type and amount for 30wt\% fiber composites

\begin{tabular}{|c|c|c|c|c|c|c|c|c|c|}
\hline \multirow{2}{*}{ Increase } & \multicolumn{3}{|c|}{ AS treatment } & \multicolumn{3}{c|}{ MS treatment } & \multicolumn{3}{c|}{ MAPP treatment } \\
\cline { 2 - 10 } & $0.5 \%$ & $1 \%$ & $2.5 \%$ & $0.5 \%$ & $1 \%$ & $2.5 \%$ & $2.5 \%$ & $5 \%$ & $10 \%$ \\
\hline CE & 2.2 & 8.8 & 0 & 0.6 & 8.3 & 2.2 & 28.0 & 32.7 & 25.1 \\
\hline SD & 2.2 & 9.4 & 0 & 1.0 & 6.0 & 1.0 & 46.1 & 50.4 & 35.8 \\
\hline WS & 14.2 & 10.2 & 8.2 & 6.8 & 11.6 & -6.2 & 51.0 & 49.4 & 49.6 \\
\hline
\end{tabular}

Young's Modulus of Composites. As seen in Fig.3, Young's Modulus tends to increase with increasing CE loading. $40 \mathrm{wt} \%$ of CE had increased young's modulus from $1243 \mathrm{MPa}$ to $3910 \mathrm{MPa}$ which corresponds to a 214\% increase. When effect of coupling agents on Young's Modulus of PP/CE composites was investigated (Fig. 4), it can be deduced that none of the coupling agents had a significant influence on Young's Modulus except for MAPP. There were fluctuations in Young's modulus of composites treated with AS or MS compared to untreated composites but these fluctuations were generally in the range of error. This is an evidence that AS and MS did not change interface or deformation capacity of the composites, thus Young's Modulus was not significantly 
affected. MAPP had a great influence on Young's Modulus of CE/PP composites, especially at high loadings. The same discussion of effect of MAPP on tensile strength is valid for the discussion of Young's Modulus. At low loadings, too high amount of MAPP had covered CE particles and interfacial strength was decreased, thus Young's Modulus of composites was not affected significantly by employment of MAPP. However, at 30 and 40wt\% CE loading, Young's Modulus increased 57.1 and $34.2 \%$ compared to untreated composites at the same loading, respectively. This is an indicator of better adhesion between fiber and matrix.

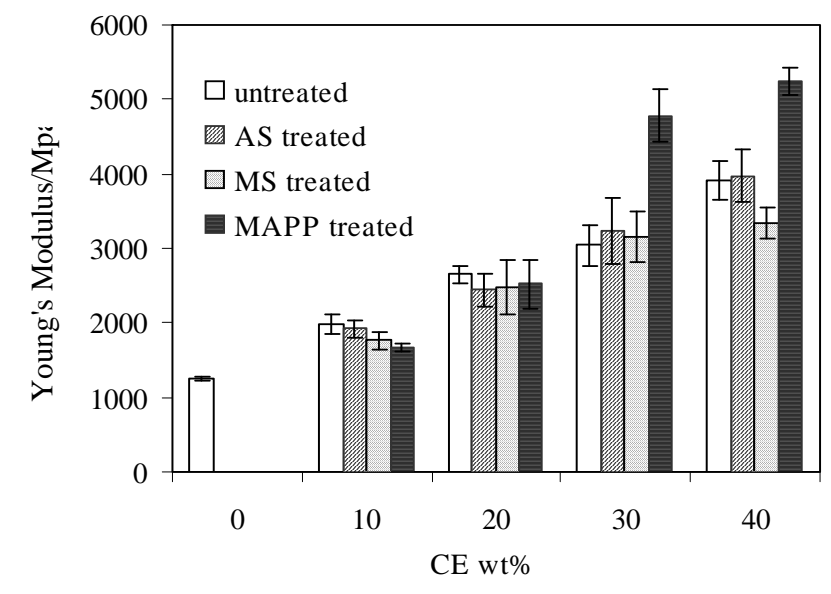

Fig.3. Effect of fiber loading and treatment type on Young's Modulus of PP/CE composites

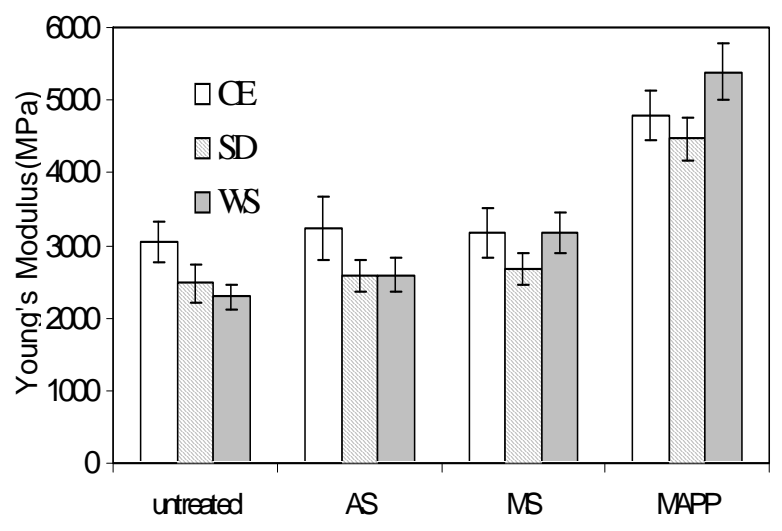

Fig.4. Effect of fiber and treatment on Young's Modulus of PP/CE, SD, WS composites at 30wt\% fiber loading

Strain at Break. Fig. 5 shows effect of fiber loading on elongation at break and energy to break of $\mathrm{PP} / \mathrm{CE}$ composites. It was observed that loading had an adverse effect on both elongation at break and energy to break. It has to be mentioned that elongation at break and energy to break of pure PP is $418 \%$ and $12 \mathrm{~N} \cdot \mathrm{m}$, respectively. Only $10 \mathrm{wt} \%$ loading of CE had a great impact on elongation and toughness of composites. Elongation at break decreased from $418 \%$ to about $8 \%$ and energy to break decreased from $12 \mathrm{~N} \cdot \mathrm{m}$ to $0.35 \mathrm{~N} \cdot \mathrm{m}$. These observations show that incorporation of particles causes a brittle behavior in composites compared to ductile thermoplastic matrix, even at low fiber loadings. This is because particles or fibers restrict deformation capacity in elastic zone as well as plastic zone. Restricted deformation capacity in the elastic zone causes increase in modulus whereas restricted deformation capacity in the plastic zone causes decreased elongation at break and toughness.

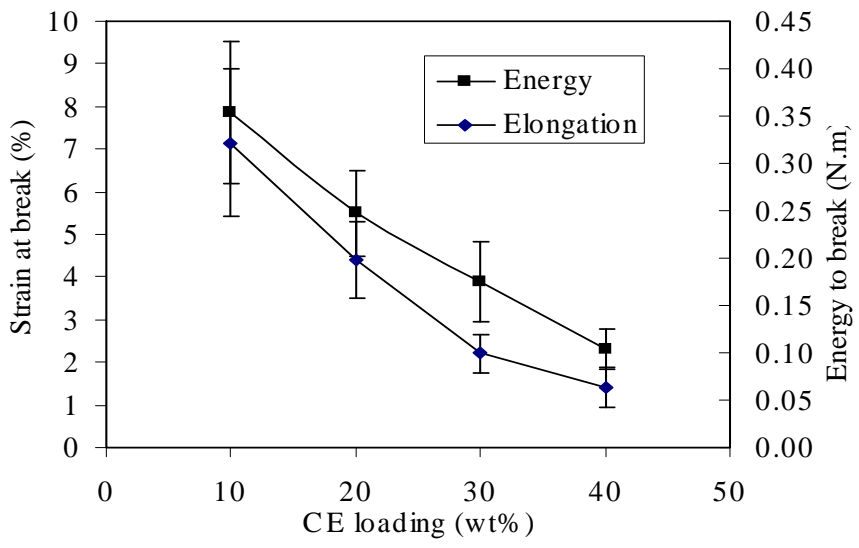

Fig.5. Effect of CE loading on strain at break and energy to break of PP/CE

Fig. 6 and Fig. 7 illustrate the deviation of strain at break and energy to break of CE, SD and WS composites with AS, MS and MAPP treatment, respectively. Considering error bars, AS and MS treatment did not change strain at break and energy to break of composites significantly for three types of composites, but MAPP significantly reduced the two responses. The reduction in strain at break was 33.5, 33.9, and $44.8 \%$ for CE, SD, and WS/PP composites, respectively. Similarly, reduction in energy to break was 10.0, 21.4 and 33.3\% for CE, SD, and WS/PP composites, 
respectively. MAPP have reduced strain at break and, consequently energy to break values of composites due to enhanced adhesion between fiber and matrix. Better adhesion yields to more restriction of deformation capacity of composites, thus catastrophic failure occurs after small strain deformations. It would be expected that silane coupling agents would decrease strain at break and toughness of composites due to enhancement of adhesion between polymer and fiber but it seems that limited enhancement of the interface was not reflected in toughness of the composites.
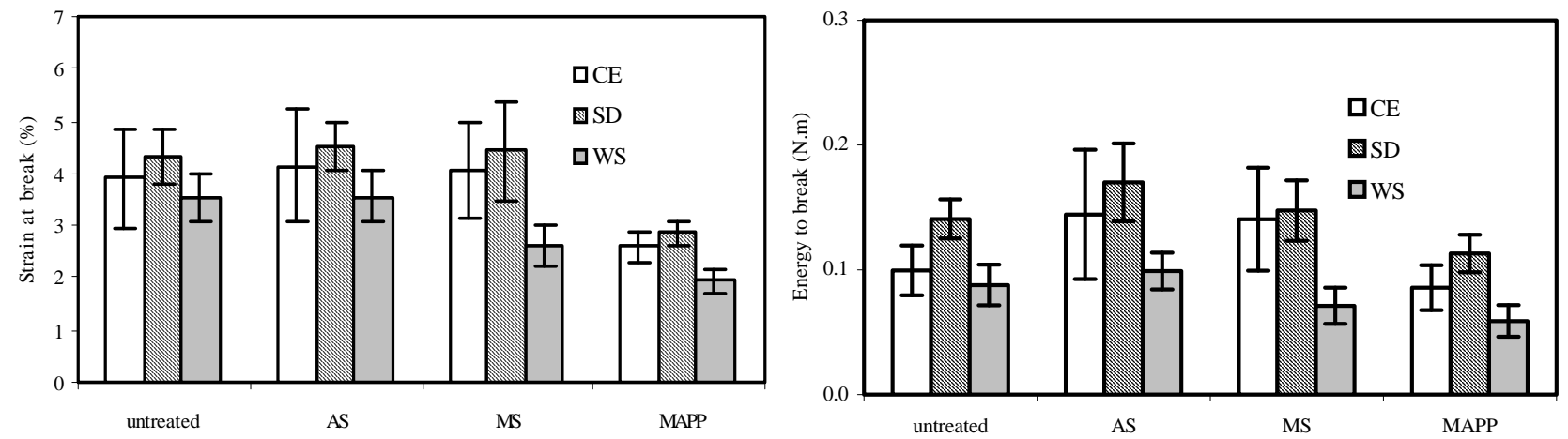

Fig.6. Effect of fiber and treatment on strain of $\mathrm{PP} / \mathrm{CE}, \mathrm{SD}$, WS composites at 30\% fiber

Fig.7. Effect of fiber and treatment on break energy of PP/CE, SD, WS composites at 30\% fiber

\section{Conclusions}

Tensile strength of the PP/fiber composites tends to decrease and Young's Modulus tends to increase with increasing fiber volume fraction. MAPP treatment exhibited the best performance especially for PP/SD composites. Young's Modulus increase in untreated composites was over 200\% and MAPP treatment provided over 50\% further increase in Young's Modulus. Optimum coupling agent concentration was found to be $1 \mathrm{wt} \%$ with respect to fiber and $5 \mathrm{wt} \%$ MAPP with respect to PP for maximum mechanical properties. MAPP treatment give rise to decline in toughness due to enhanced interactions between fiber and matrix whereas silane treatment did not have a significant effect on toughness of PP/fiber composites. In the light of these measurements, interfacial interactions, thus stress transfer between fiber and matrix, were considerably improved via MAPP treatment. Silane treatments also had the same effect but to a lesser extend.

\section{Acknowledgements}

This work was financially supported by Taizhou Science \& Technology Project (Grant No. 14GY02) and Maoming Science \& Technology Project (Grant No. 915325).

\section{References}

[1] G. Caprino, L. Carrino, M. Durante: Composite Structures Vol.133 (2015), p. 892

[2] H. Mechakra, A. Nour, S. Lecheb, A. Chellil: Composite Structures Vol.124 (2015), p. 152

[3] O.M.L. Asumani, R.G. Reid, R. Paskaramoorthy: Composites Part A Vol. 43(2012), p. 1431

[4] C.A. Juarez, G. Fajardo, S. Monroy: Construction and Building Materials Vol. 91(2015), p. 164

[5] Faris M. AL-Oqla, S.M. Sapuan: Synthetic Metals Vol. 206(2015), p.42

[6] L.Q.N.Tran, T.Nguyen Minh, C.A. Fuentes: Industrial Crops and Products Vol. 65(2015), p. 437

[7] M.N. Ichazo, C. Albano, J. Gonzales, R. Perera, M.V. Candal: Composite Structures Vol.54 (2001), p. 207 